WILEY-VCH

\title{
Individualized p-Doped Carbon Nanohorns
}

\author{
Anastasios Stergiou, ${ }^{[\mathrm{a}]}$ Zheng Liu, ${ }^{[\mathrm{b}],[\mathrm{cc}]}$ Bin Xu, ${ }^{[\mathrm{d}]}$ Toshiro Kaneko, ${ }^{[\mathrm{d}]}$ Christopher P. Ewels, ${ }^{[\mathrm{e}]}$ Kazu \\ Suenaga,${ }^{[c]}$ Minfang Zhang, ${ }^{[c]}$ Masako Yudasaka, ${ }^{[c]}$ and Nikos Tagmatarchis ${ }^{\star[a]}$
}

\begin{abstract}
A facile approach to individualize spherically aggregated pristine carbon nanohorns (pr-CNHs) was established. Specifically, we found that treatment of pr-CNHs with chlorosulfonic acid generates positively charged polarized species, which disintegrate toward individualized carbon nanohorns (in-CNHs). Interestingly, the isolated in-CNHs unveiled to be p-doped owed to the adsorption of chlorosulfonate units. The findings were justified and proved by data derived from high-resolution transmission electron microscopy imaging, Raman and ultraviolet photoemission spectroscopy, while additionally supported by theoretical calculations and thermogravimetry.
\end{abstract}

Individualization of synthetic carbon allotropes, i.e. carbon nanotubes (CNTs), graphene, and carbon nanohorns (CNHs), is critical for attaining full advantage of their novel properties. For example, the characteristic photoluminescence of single-walled CNTs (SWCNTs) can be observed only in debundled material, particularly in isolated SWCNTs, ${ }^{[1]}$ while graphene (1-10 layers), a well known zero band-gap semiconductor, emits light under femtosecond pump-probe excitation unlike graphite stacks. ${ }^{[2]}$ Another important parameter for the better applicability of synthetic carbon allotropes is to keep the individualized species intact from chemical and structural defects. Incorporation of defects on the $\mathrm{sp}^{2}$-hybridized carbon lattice disrupts the extended $\pi$-conjugation and lowers conductivity and mechanical strength. ${ }^{[3]}$ Defects mainly originate from solvents and/or gases dissolved in those solvents employed en route for debundling and/or exfoliation, ${ }^{[4]}$ while application of mechanical stretch leads to cracking of the graphitic lattice. ${ }^{[3]}$ Additionally, heteroatoms such as hydrogen, oxygen and nitrogen are often inserted within the graphitic lattice, hence, highly pure defect-free materials remain a challenge for organic electronics and biological applications.

Ideally, CNHs are considered as monolayered graphene wrapped into a long conical tip. They are encompassed of $\mathrm{sp}^{2}$ hybridized carbon atoms, forming closed nanocages with 2-5 nm

[a] A. Stergiou, Dr. N. Tagmatarchis Theoretical and Physical Chemistry Institute, National Hellenic Research Foundation 48 Vassileos Constantinou Avenue, Athens 11635 (Greece) E-mail: tagmatar@eie.gr

[b] Dr. Z. Liu

Inorganic Functional Materials Research Institute, National Institute of Advanced Industrial Science and Technology (AIST) Anagahora 2266-98, Shimoshidami, Moriyamaku, Nagoya 463-8560 (Japan)

[c] Dr. Z. Liu, Dr. K. Suenaga, Dr. M. Zhang, Dr. M. Yudasaka Nanomaterials Research Institute, National Institute of Advanced Industrial Science and Technology (AIST)

Central 5, 1-1-1 Higashi, Tsukuba 305-8565 (Japan)

[d] Dr. B. Xu, Dr. T. Kaneko

Department of Electronic Engineering, Tohoku University 6-6-05 Aoba, Aramaki, Aoba-Ku, Sendai 980-8579 (Japan)

[e] Dr. C. P. Ewels

Institut des Materiaux Jean Rouxel, CNRS, Universite de Nantes 2 Rue de la Houssiniere, BP32229, 44322 Nantes (France)

Supporting information for this article is given via a link at the end of the document. in diameter and 20-50 nm in length. Pristine $\mathrm{CNHs}$ assemble in spherical clusters with size $80-100 \mathrm{~nm}$ in diameter, during production via laser ablation of graphite. Structurally, CNHs contain not only hexagonal but also pentagonal and heptagonal rings and this particular motif resulted in a rich and varied chemistry. ${ }^{[5]}$ Besides the enhanced reactivity associated with decrease in aromaticity at defects, local chemical reactivity is also increased at regions of higher curvature of $\mathrm{CNH}$ due to pyramidal distortion of the $s p^{2}$ carbon hybridization. ${ }^{[6]}$ Importantly, CNHs offer as key advantage over CNTs the absence of metal catalyst in their synthesis. Specifically, the presence of metal particles in the production of CNTs necessitates additional treatment with strong acids, a detrimental process for the graphitic network due to the incorporation of defects. Contrary, pure $\mathrm{CNHs}$ are easily accessible, better facilitating the study and understanding of their properties and applications. Nonetheless, the research and development of $\mathrm{CNH}$ has been decelerated by their aggregation, rendering dispersion and dismantling difficult, hence prohibiting processing of individualized species and limiting their immediate utility particularly in bioapplications.

Without surprise, individualization of $\mathrm{CNH}$ is critical and in this direction smaller aggregates were initially obtained upon dispersion of pristine $\mathrm{CNHs}$ in deuterated sodium dodecyl benzene sulfonate followed by ultracentrifugation. ${ }^{[7]}$ Later on, sucrose density gradient centrifugation of oxidized $\mathrm{CNHs}$, dispersed by sodium cholate, allowed the isolation of low-yield fractions of individualized CNHs, in a virtually non-scalable method, thus non-applicable for bulk production. ${ }^{[8]}$ Recently, some of us succeeded the dismantling and individualization of $\mathrm{CNHs}$ by the reductive dissolution of the raw aggregates in the presence of potassium naphthalenide under an inert atmosphere. ${ }^{[9]}$ However, in the latter methodology, the essential requirement of a glove box, not only for performing the processing en route for dismantling $\mathrm{CNHs}$, but also for protecting the reduced material from air and moisture, is itself a serious drawback, particularly for handling the material. Significantly, in all those three approaches for individualizing $\mathrm{CNHs}$, the final material was obtained functionalized and accompanied by amorphous graphitic particles.

Carbon nanotubes, graphene and $\mathrm{C}_{60}$ are weak Brönsted bases and protonation of the graphitic lattice takes place under treatment with a strong Brönsted acid. Considering that superacids, stronger than $100 \%$ sulfuric acid, act as proton donors, SWCNTs and $\mathrm{C}_{60}$ can be converted to protonated nanocarbon derivatives upon superacid treatment. ${ }^{[10]}$ In fact $\mathrm{HC}_{60}{ }^{+}$carbocation has been successfully isolated in solid state via treatment into an artificial superacid. ${ }^{[10]}$ The highly symmetric carbon cage offers efficient electronic stabilization of the carbocation, through delocalization of the positive charge. In contrast to $\mathrm{C}_{60}$, nuclear magnetic resonance studies on SWCNTs mixed with commercial superacids propose the polarization of the carbon lattice due to the highly electrophilic protons of the superacid, instead of a direct protonation mechanism. ${ }^{[11]}$

Motivated by the aforementioned points, we succeeded in the individualization and isolation of intact $\mathrm{CNHs}$, without being structurally modified, in bulk quantities, i.e. gram scale. Herein, a 
scalable process based on superacid treatment of spherically aggregated $\mathrm{CNHs}$ and the isolation of individualized nanohorns is reported. The individualization process initiates via the polarization of the $s p^{2}$ lattice of pristine $\mathrm{CNHs}$ by the proton of the superacid, in a similar process to the one reported for SWCNTs. Dispersions of individualized nanohorns with concentration higher than $2 \mathrm{mg} / \mathrm{mL}$ are stable for more than a year. Raman spectroscopy, high-resolution transmission electron microscopy (HR-TEM) imaging and ultraviolet photoelectron spectroscopy (UPS), complemented by theoretical calculations, modeling and thermogravimetry, delivered meaningful information in terms of nature and quality for the nanohorn species isolated. Importantly, the individualized material displays a p-doped behavior, arising from adsorbed chlorosulfonate anions onto the graphitic skeleton (see below).

The dismantling process of pristine $\mathrm{CNHs}$, abbreviated as pr-CNHs, was carried out upon mixing with chlorosulfonic acid under mild sonication. Similarly to other graphitic nanocarbon materials, chlorosulfonic acid interacts electrostatically with $\mathrm{CNHs}$ without prompting chemical oxidation. In this sense, the highly electrophilic protons of the acid polarize the $\mathrm{sp}^{2}$-carbon network generating positively charged nanocarbon species, abbreviated as chr-CNHs, and initiate electrostatic repulsive forces within the spherically aggregated cluster, thereby, inducing disintegration toward individualized nanohorn species, abbreviated as in-CNHs. Ultimately, addition of water in the reaction system hydrolyses the superacid and precipitates the in-CNHs. Additionally, the local heat and gaseous $\mathrm{HCl}$ generated upon the exothermic breakdown of chlorosulfonic acid by water trigger further the individualization process.

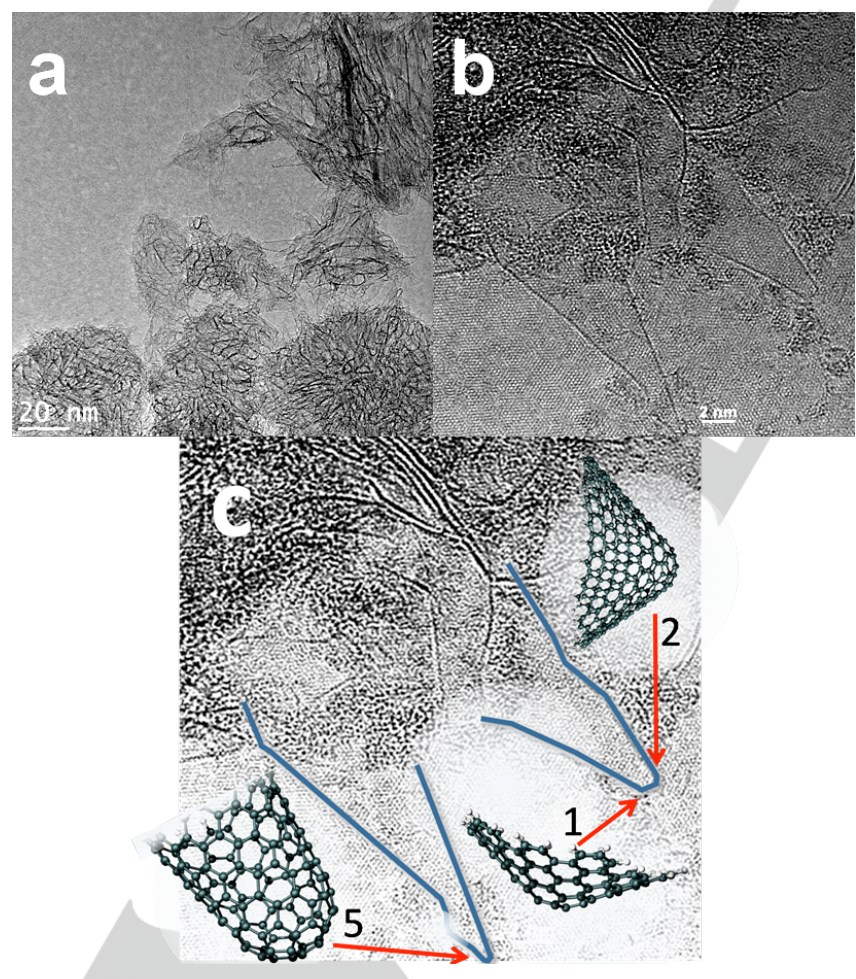

Figure 1. Representative HR-TEM images for (a) pr-CNHs, and (b) isolated in-CNHs. (c) Schematic highlighting the presence of the two in-CNHs shown in Fig. 1b, with possible atomic scale structures at different points around the cone tips indicating the number of pentagons corresponding to the change in observed angle.

The individualization of CNHs is strongly supported by HR-TEM imaging. Specimens for imaging were prepared by dispersing the material in methanol and dropping it onto Mo micro grids covered by monolayered boron nitride (BN) film. The particular grid offers the advantage of supporting the in-CNHs, which are clearly visible with distinct contrast on the monolayered $\mathrm{BN}$ substrate, even though the lattice of $\mathrm{h}-\mathrm{BN}$ is also visible underneath the contrast of in-CNHs. In Fig. 1, HR-TEM images of pr-CNHs and in-CNHs are compared. While only spherical superstructures due to $\mathbf{p r}$-CNHs were visualized under the microscope, superacid treatment of pr-CNHs resulted in the breakdown of those aggregates, yielding in-CNHs, imaged as single conical tubules with diameter 2-5 nm and length 20-40 nm (see also Supporting Information, Fig. S1). Furthermore, the size distribution of in-CNHs was measured by dynamic light scattering (DLS), a powerful tool to analyze dilute dispersions of materials and obtain information about their size in liquid phase. Comparing with DLS assays performed on pr-CNHs in DMF, it is found that the apparent hydrodynamic radius of in-CNHs, calculated by employing cumulant analysis, was smaller as compared to that owed to pr-CNHs (i.e. $50 \mathrm{~nm}$ vs $100 \mathrm{~nm}$ ). The smaller size for in-CNHs vs pr-CNHs identified in the DLS studies is in accordance with the HR-TEM findings, particularly with the presence of individualized species. However, one should keep in mind that the actual values for the hydrodynamic radius as estimated by DLS cannot be directly compared with the size of species imaged by HR-TEM, since the former reflect interactions with solvent in liquid phase, which are obviously absent when performing HR-TEM imaging in the solid state, while also depend on the shape of the dispersed nanostructure, i.e. in-CNHs possess elongated conical morphology, in contrast to spherical pr-CNHs.

UV-Vis spectroscopy assays of as prepared dispersions of $\mathrm{CNHs}$ in chlorosulfonic acid, chr-CNHs, do not reveal any new spectral features (Supporting Information, Fig. S2), unlike SWCNTs and $\mathrm{C}_{60}$, where the protonated nanocarbon materials exhibit an absorption peak at $800-1000 \mathrm{~nm}$ corresponding to insitu generated carbocationic species. ${ }^{[1], ~[10] ~ M o r e o v e r, ~ t h e ~}$ electronic absorption spectrum of in-CNHs remains featureless, likewise the UV-Vis spectrum of pr-CNHs.

In the Raman spectrum of pr-CNHs, two characteristic bands are present (Fig. 2), the G-band located at $1574 \mathrm{~cm}^{-1}$ and assigned to the $E_{2 g}$-like vibrations within the $\mathrm{sp}^{2}$ hybridized carbon atoms and the D-band ascribed to the $\mathrm{A}_{1 \mathrm{~g}}$-symmetry modes located at $1335 \mathrm{~cm}^{-1}$ and attributed to the loss of the basal symmetry. The D-band in the Raman spectrum of inCNHs was identical to the one owed to pr-CNHs, demonstrating that no chemical oxidation, via introduction of oxo-functionalities onto the graphitic framework, took place under the chlorosulfonic acid treatment, proving that the composition of the lattice was maintained. ${ }^{[12]}$ As already discussed, it is well established that treatment of $\mathrm{C}_{60}$, SWCNTs and graphene with superacids either directly protonates (i.e. $\mathrm{C}_{60}$ ) or polarizes (i.e. SWCNTs and 
graphene) the $\mathrm{C}=\mathrm{C}$ bonds in a reversible and non-oxidative manner. ${ }^{[10],[12]}$

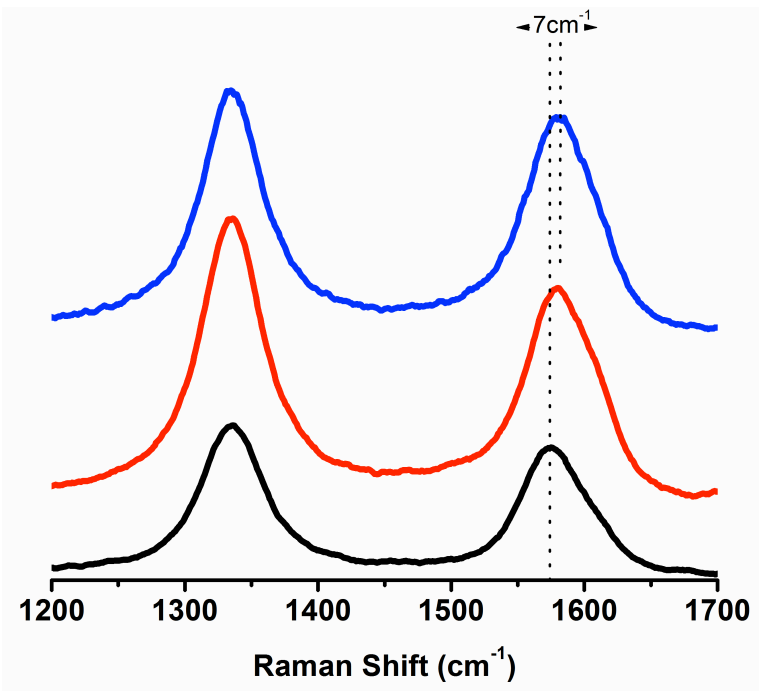

Figure 1. Raman spectra for pr-CNHs (black), chr-CNHs (blue) and in-CNHs (red), obtained upon excitation at $514 \mathrm{~nm}$.

The solubilization of carbon allotropes in strong Brönsted acids arise from a charge-transfer process from the valence band of the nanocarbon material to the highly electrophilic protons of the superacid. Careful examination of the G-band position in prCNHs and in-CNHs reveals that treatment of pr-CNHs with chlorosulfonic acid resulted in $7 \mathrm{~cm}^{-1}$ shift of the G-band to higher wavenumbers. Considering the high sensitivity of G-band to electronic doping effects, the latter blue shift identified in inCNHs is directly related to loss of electrons from the valence band, namely to $p$-doping of the carbon lattice. ${ }^{[13]}$ We propose that the precursors of in-CNHs - the species that exist before the aqueous quenching - are ion pairs consisting of positively charged $\mathrm{CNHs}$ and chlorosulfonate counter ions. Importantly, the Raman study for chr-CNHs revealed an enhancement of the $\mathrm{D} / \mathrm{G}$ ratio by ca. $30 \%$ as compared to that due to pr-CNHs, which is restored in in-CNHs (Fig. 2). The amplification of the D/G ratio in chr-CNHs is driven by the extended polarization of the $\mathrm{sp}^{2}$ lattice of nanohorns by chlorosulfonic acid. Hence, the decrease of electron density of the valence band in pr-CNHs enriches their $\mathrm{sp}^{3}$ nature against $\mathrm{sp}^{2}$, thereby, inducing the enhancement of the D/G ratio. Likewise in in-CNHs, the G-band of chr-CNHs was similarly found blue-shifted by $7 \mathrm{~cm}^{-1}$ as compared to that owed to pr-CNHs, proving that the p-doping process initiates at that stage and not during the quenching with water. Moreover, the addition of water into the superacid surrounded ion pairs of chr-CNHs hydrolyses the large excess of chlorosulfonic acid, prompting precipitation of in-CNHs. The aforementioned mechanism based on the Raman findings is graphically illustrated on Scheme 1.

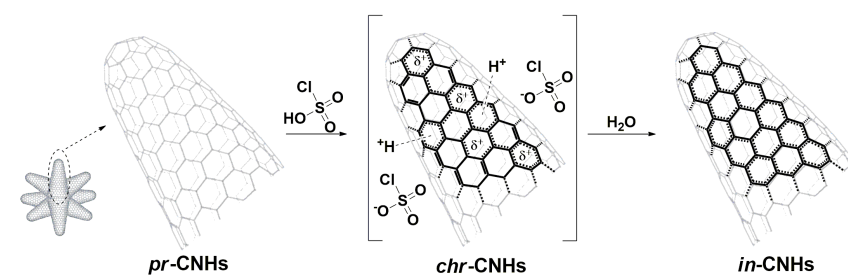

Scheme 1. Polarization of the $\mathrm{sp}^{2}$ skeleton of $\mathrm{pr}$-CNHs upon chlorosulfonic acid treatment inducing positive charges which eventually due to electrostatic repulsive forces result on the generation of in-CNHs.

The p-doping of in-CNHs is further verified by ultraviolet photoelectron spectroscopy. Figure 3 shows the ultraviolet photoemission spectra of pr-CNHs and in-CNHs at ambient conditions. The energy difference between the highest occupied molecular orbital level and the vacuum level, which corresponds to the ionization potential, is estimated by fitting a straight line and determining its intersect with the energy axis. It is found that the ionization potential of pr-CNHs is $4.7 \mathrm{eV}$. On the other hand, the ionization potential of in-CNHs is $4.8 \mathrm{eV}$, higher than that of pr-CNHs, justifying that the material is p-doped.
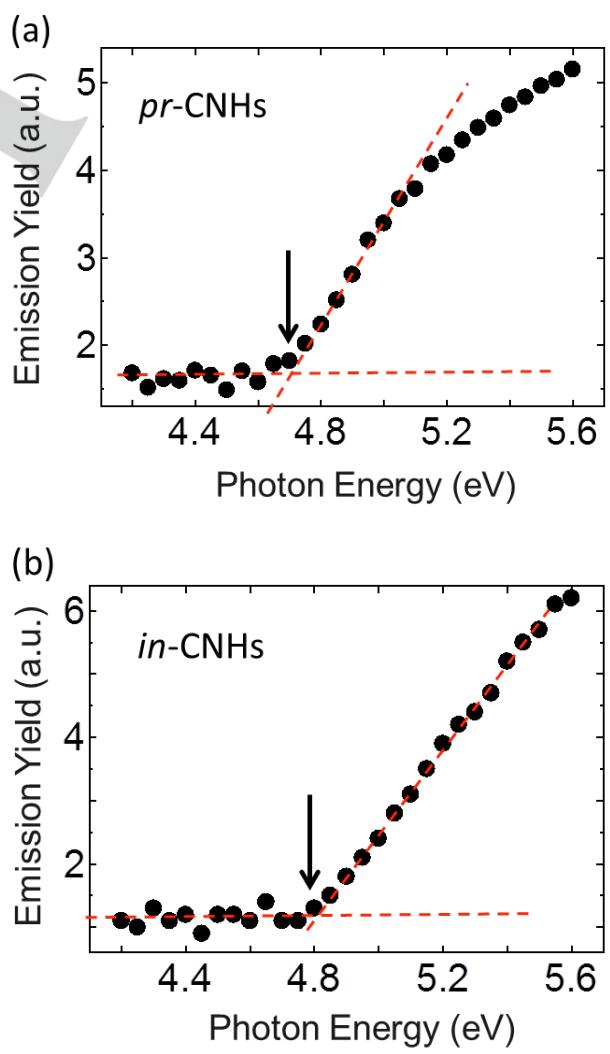

Figure 3. Ultraviolet photoemission spectra for (a) pr-CNHs, and (b) in-CNHs at ambient conditions. The arrows show the HOMO level, which corresponds to the ionization potential. 
Density-functional (local density approximation) calculations ${ }^{[14]}$ were employed to explore the interaction of chlorosulfonate anions with both graphene (using a 128-carbon atom hexagonal $8 \times 8$ unit cell supercell with a $2 \times 2 \times 1$ k-point grid) and a 5pentagon nanohorn $\left(\mathrm{C}_{153} \mathrm{H}_{15}\right)$. The charge density was fitted using plane waves, with wave functions constructed using $18 / 28 / 40 / 40$ independent Gaussian based functions used for $\mathrm{C} / \mathrm{S} / \mathrm{Cl} / \mathrm{O}$ with angular momentum up to $\mathrm{I}=2$, and electron temperature of $\mathrm{kT}=0.01 \mathrm{eV}$. The chlorosulfonate anion preferentially arranges itself above a pentagon of the nanohorn tip with two oxygen atoms above neighbouring carbon atoms and $\mathrm{Cl}$ above the opposite carbon (Figure 4a). A charge transfer of $0.77 \mathrm{e}$ is seen from the nanohorn tip to the anion. In contrast the anion binding is $0.288 \mathrm{eV}(6.64 \mathrm{kcal} / \mathrm{mol})$ stronger to graphene, with a $0.91 \mathrm{e}$ transfer. This suggests preferential binding of the anion to the nanohorn sidewalls rather than tips.

We can use the calculated charge transfer and experimental Raman G-band shift to estimate the surface coverage of chlorosulfonate anions. Calculations of the G-band frequency for graphene were performed on a 2-atom graphene unit cell with a $24 \times 24 \times 1 \mathrm{k}$-point grid, 38 functions per carbon and finite electron temperature of $k T=0.04 \mathrm{eV}$. Figure $4 \mathrm{~b}$ shows the frequency shift for a graphene sheet as a function of charge state. Considering curvature effects as negligible these frequencies can be compared directly to the experimental Gband Raman modes of the nanohorns. Within the range of experimental error they agree well with the observation that prCNHs begin typically n-type $\left(1574 \mathrm{~cm}^{-1}\right)$ and after separation to their individual components, in-CNHs shift towards p-type (1581 $\mathrm{cm}^{-1}$ ). This $7 \mathrm{~cm}^{-1}$ shift, from Fig. $4 \mathrm{~b}$ implies a charge-transfer of 0.0055 electrons per carbon atom during this process, thus with our calculated charge transfer of 0.91 e per chlorosulfonate anion, this implies one chlorosulfonate anion per every 140 carbon atoms.

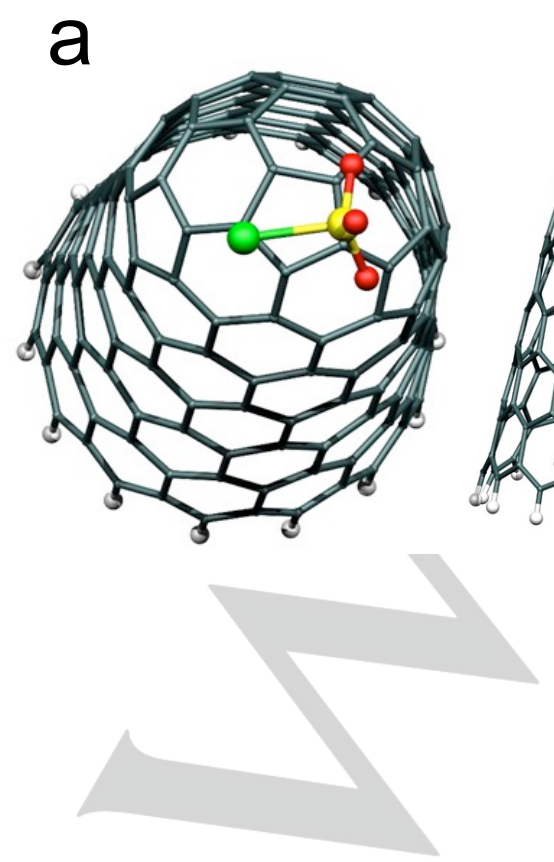

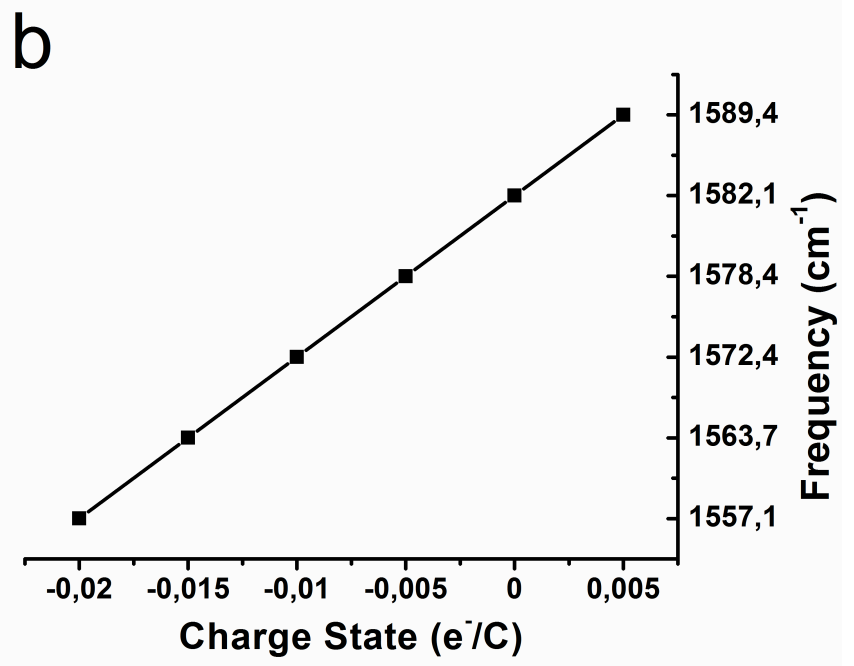

Figure 4. (a) DFT optimized structure of $\mathrm{ClSO}_{3}$ at the tip of 5-pentagon inCNH $\left(\mathrm{C}_{153} \mathrm{H}_{15}\right)$; top view (left) and side view (right). The distance to the nearest carbon atom in the nanohorn tip is: $2.44 \AA$ and $2.66 \AA$ (two O atoms), $2.96 \AA$ (Cl) and $3.27 \AA$ (S). (b) Calculated variation in G-band frequency $\left(\mathrm{cm}^{-1}\right)$ for a graphene sheet as a function of graphene charge state (electrons per carbon atom). The best fit line corresponds to the formula $y=(1272.6 x+1583.4) \mathrm{cm}^{-1}$.

Next, dilute DMF dispersions of pr-CNHs and in-CNHs were drop-casted onto a glassy carbon electrode (GCE) and with the aid of cyclic voltammetry we found that the current was increased due to the higher conductivity of nanohorns (Supporting Information, Fig. S3). Moreover, from CV assays conducted in the presence of $\mathrm{Fc} / \mathrm{Fc}^{+}$, which can be reversibly oxidized under an applied potential, it was deduced that Fc oxidizes easier as compared to the bare GCE for both pr-CNHs and in-CNHs (Supporting Information, Fig. S4).

Finally, the thermal stability of in-CNHs was examined by thermogravimetric analysis (TGA) conducted under nitrogen atmosphere. The exceptional thermal stability of $\boldsymbol{p r}$-CNHs, up to $900{ }^{\circ} \mathrm{C}$ under inert conditions was initially proved. However, when in-CNHs examined, a weight loss in the temperature range $195-250{ }^{\circ} \mathrm{C}$ was observed (Fig. 5). Considering that (a) inCNHs were vacuum dried $\left(100{ }^{\circ} \mathrm{C}, 0.3\right.$ bar, $12 \mathrm{hrs}$ ) before conducting the TGA assays, and (b) covalently functionalized $\mathrm{CNHs}$ thermally decompose at $250-500{ }^{\circ} \mathrm{C}$ by first losing the attached organic addends, ${ }^{[15]}$ the relatively lower temperature thermal decomposition identified for in-CNHs is assigned to adsorbed residual chlorosulfonate-based species. This is further corroborated by considering that sulfonated resins decompose above $220{ }^{\circ} \mathrm{C},{ }^{[16]}$ affirming that if sulfonate moieties were covalently anchored on in-CNHs their decomposition would have been observed above that temperature. However, the first derivative of the TGA curve for in-CNHs shows a major thermal degradation step at $196{ }^{\circ} \mathrm{C}$, a temperature far above the boiling point of trapped sulfuric acid produced during the hydrolysis of chlorosulfonic acid or water molecules and low enough to the one assigned to covalently attached sulfonate functionalities. 


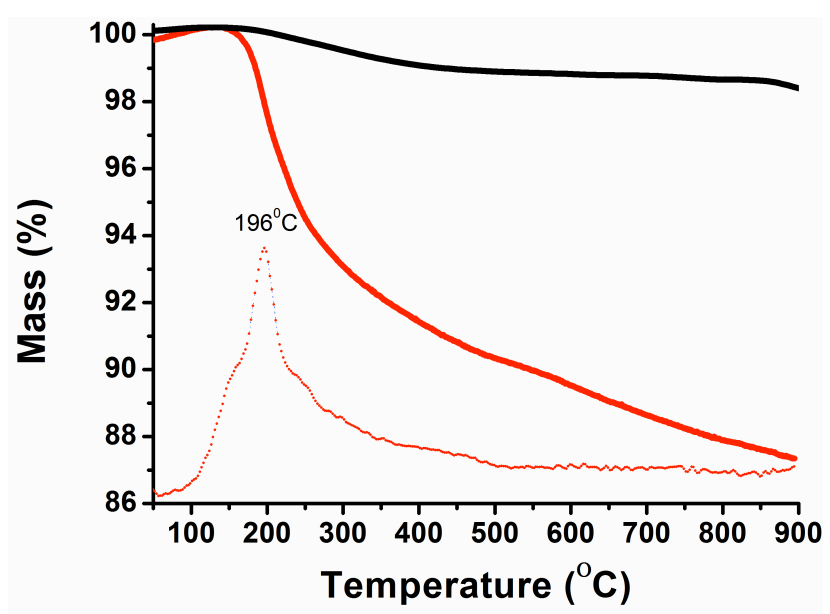

Figure 5. Thermographs for pr-CNHs (black) and in-CNHs (red), obtained under inert atmosphere. The first derivative of the thermogravimetral curve for in-CNHs is also shown (red dotted).

The information derived by TGA, referring to the presence of residual chlorosulfonate species adsorbed on in-CNHs, is additionally supported by attenuated-total-reflectance infrared spectroscopy (ATR-IR). The ATR-IR spectrum of in-CNHs resembles that of pr-CNHs, both dominated by the lack of vibrations due to the absence of any oxo-functionalities (i.e. -C$\mathrm{O}-\mathrm{C}-,-\mathrm{COOH})$, hence, validating the non-oxidative superacid treatment. The evolution of two weak broad spectral features around $1050 \mathrm{~cm}^{-1}$ and $1250 \mathrm{~cm}^{-1}$ (Supporting Information, Fig. $\mathrm{S} 5)$, assigned to symmetric and asymmetric stretching vibrations of chlorosulfonate anions, ${ }^{[17]}$ proves that those species are adsorbed onto the carbon framework of in-CNHs.

In conclusion, a facile approach for the individualization of carbon nanohorns was developed. Until now, procedures en route for obtaining in-CNHs suffer from serious drawbacks, such as handling the material in an air-and-moisture protected environment, while they are also non-scalable. We showed that treatment of spherically aggregated pr-CNHs with chlorosulfonic acid, in a scalable process to gram amounts, yields high quality of $\mathbf{p r}$-CNHs. The dismantling process involves polarization of the $s p^{2}$ lattice of $p r-\mathrm{CNHs}$ by the proton of the superacid, without prompting chemical oxidation, followed by electrostatic repulsive forces developed within the aggregated cluster, eventually yielding in-CNHs. More importantly, adsorption of chlorosulfonate species onto in-CNHs resulted in p-doping, which was proved by Raman and ultraviolet photoemission spectroscopy and additionally supported by theoretical calculations. We are currently examining the chemistry and reactivity of those in-CNHs, particularly toward the preparation of functional materials for organic electronics and solar cells and we strongly believe that new avenues for this synthetic carbon allotrope are awaiting exploration.

\section{Experimental Section}

Preparation of in-CNHs: In a round bottom flask, chlorosulfonic acid (100 $\mathrm{mL}$ ) was added to $\mathrm{pr}$-CNHs $(200 \mathrm{mg})$, resulting to rapid dissolution and formation of an ink-like homogeneous solution, and the system was bath sonicated for $2 \mathrm{hrs}$. To isolate in-CNHs in a powder form, the superacid was carefully quenched under stirring with deionized water [EXTREME CARE SHOULD BE TAKEN. The reaction of chlorosulfonic acid with water is extremely exothermic followed by evolution of gaseous $\mathrm{HCl}$. The quenching procedure must be performed in fume hood.]. The in-CNHs precipitated as the quenching proceeds. The addition of water continued until no $\mathrm{HCl}$ evolution was observed. The hot mixture allowed reaching room temperature, then filtered through a PTFE membrane filter $(0.1 \mu \mathrm{m}$ pore size) and finally extensively washed with water, methanol and dichloromethane to remove acid and water residues. Afterwards, inCNHs vacuum dried $\left(100{ }^{\circ} \mathrm{C}, 0.3 \mathrm{bar}, 12 \mathrm{hrs}\right)$ and collected as a black powder, which gave stable dispersions in methanol. The procedure can easily be carried out in gram scale with a conventional cleaning sonic bath.

\section{Acknowledgements}

This project has received funding from the European Union's Horizon 2020 research and innovation programme under the Marie Sklodowska-Curie grant agreement No 642742. ZL and KS acknowledge financial support from MEXT-KAKENHI (25107003) and JST-ACCEL.

Keywords: carbon nanohorns $\cdot$ individualization $\cdot$ chlorosulfonic acid $\cdot$ p-doping $\cdot$ electronic properties

[1] M. O'Connell, S. Bachilo, C. Huffman, V. Moore, M. Strano, E. Haroz, K. Rialon, P. Boul, W. Noon, C. Kittrell, J. Ma, R. Hauge, R. Weisman, R. Smalley, Science 2002, 593, 593-596.

[2] R. Newson, J. Dean, B. Schmidt, H. M. van Driel, Opt. Express 2009, 17, 2326-2333

[3] F. Banhart, J. Kotakoski, A. Krasheninnikov, ACS Nano 2011, 1, 24-41.

[4] T. Skaltsas, X. Ke, C. Bittencourt, N. Tagmatarchis, J. Phys. Chem. C 2013, 117, 23272-23278.

[5] N. Karousis, I. Suarez-Martinez, C. Ewels, N. Tagmatarchis, Chem. Rev. 2016, doi: 10.1021/acs.chemrev.5b00611

[6] S. Park, D. Srivastava, K. Cho, Nano Lett. 2003, 3, 1273-1277.

[7] M. Zhang, M. Yudasaka, J. Miyawaki, J. Fan, S. lijima, J. Phys. Chem. B 2005, 109, 22201-22204.

[8] M. Zhang, T. Yamaguchi, S. lijima, M. Yudasaka, J. Phys. Chem. C 2009, 113, 11184-11186.

[9] D. Voiry, G. Pagona, E. Canto, L. Ortolani, V. Morandi, L. Noe, M. Monthioux, N. Tagmatarchis, A. Penicaud, Chem. Commun. 2015, 51, 5017-5019.

[10] a) S. Ramesh, L. Ericson, V. Davis, R. Saini, C. Kittrell, M. Pasquali, W. Billups, W. Adams, R. Hauge, R. Smalley, J. Phys. Chem. B 2004, 108, 8794-8798; b) C. Reed, K. Kim, R. Bolskar, L. Mueller, Science 2000, 289, 101-104

[11] C. Engtrakul, M. Davis, T. Gennett, A. Dillon, K. Jones, M. Heben, J. Am. Chem. Soc. 2005, 127, 17548-17555.

[12] S. Bandow, A. Rao, G. Sumanasekera, P. Eklund, F. Kokai, K. Takahashi, M. Yudasaka, S. lijima, Appl. Phys. A-Mater. Sci. Process. 2000, 71, 561-564.

[13] N. Jaggi, S. Dhall, Fullerenes, Nanotubes and Carbon Nanostr. 2015, 23, 942-946.

[14] a) P. R. Briddon, M. J. Rayson, Phys. Stat. Solidi B: Basic Solid State Res. 2011, 248, 1309; b) M. J. Rayson, P. R. Briddon, Phys. Rev. B 2009, 80, 205104.

[15] a) G. Pagona, N. Karousis, N. Tagmatarchis, Carbon 2008, 46, 604$610 ;$ b) G. Rotas, A. S. D. Sandanayaka, N. Tagmatarchis, T. Ichihashi, M. Yudasaka, S. lijima, O. Ito, J. Am. Chem. Soc. 2008, 130, 47254731.

[16] D. Smith, J. Polymer Sci. Part C: Polymer Lett. 1966, 4, 215-221. 
[17] J. Ciruna, E. Robinson, Canad. J. Chem. 1968, 46, 1715-1718. 
WILEY-VCH

A facile approach for the individualization of aggregated carbon nanohorns, by treatment with chlorosulfonic acid, leading to $\mathrm{p}$-doped species, is demonstrated.
Anastasios Stergiou, Zheng Liu, Bin Xu, Toshiro Kaneko, Christopher P. Ewels, Kazu Suenaga, Minfang Zhang, Masako Yudasaka and Nikos Tagmatarchis*

Page No. - Page No.

Individualized p-Doped Carbon Nanohorns 